\title{
Spatial clusters of HIV-1 genotypes in a recently infected population in Yunnan, China
}

Min Chen ${ }^{\dagger}$, Yanling Ma ${ }^{\dagger}$, Huichao Chen, Jie Dai, Hongbing Luo, Chaojun Yang, Lijuan Dong, Xiaomei Jin, Min Yang, Li Yang, Lijun Song, Zhizhong Song and Manhong Jia*

\begin{abstract}
Background: As a gateway for HIV-1 in China, Yunnan has experienced dramatic changes in HIV-1 epidemics, during which HIV-1 genotypes have become complex. To track dynamic changes in HIV-1 genotypes, an HIV-1 molecular epidemiological study was implemented in the recently infected population in Yunnan.

Methods: From 6,357 HIV-1-positive samples diagnosed during the first half of 2015 in Yunnan, 586 samples were identified as recent infections with BED-capture enzyme immunoassay (CEIA) and were subjected to phylogenetic analyses. Spatial scanning analyses for the main HIV-1 genotypes were also performed.

Results: Among the 439 specimens successfully genotyped, more than ten genotypes were detected, including CRF08_BC (45.3\%), CRF07_BC (19.4\%), unique recombinant forms (URFs) (18.2\%), CRF01_AE (11.4\%), subtype C (2.1\%), CRF85_BC (1.1\%), CRF55_01B (0.9\%), subtype B (0.5\%), CRF64_BC (0.5\%), CRF59_01B (0.2\%), CRF83_cpx (0.2\%) and CRF87_cPx (0.2\%). Females, Chinese, heterosexual contact and intravenous drug injection were significantly associated with CRF08_BC infection; homosexual contact was significantly associated with CRF01_AE and CRF07_BC infection; males and non-Chinese had a higher risk of URF infection than females. Among all HIV-1 genotypes, the geographic coverage of CRF08_BC was the largest. For CRF08_BC, CRF07_BC, URFs and CRF01_AE, spatial clusters were detected. The two CRF08_BC clusters and one URF cluster were associated with heterosexual transmission, and two of CRF01_AE clusters were associated with homosexual transmission. Transmitted drug resistance (TDR)associated mutations were detected in $2.4 \%$ of individuals.

Conclusions: The diversity of HIV-1 genotypes increased in recent infections because of a long-term HIV-1 epidemic in Yunnan. The predominant HIV-1 strains showed distinct demographic characteristics and formed spatial clusters. These findings improved our understanding of the evolution of HIV-1 in Yunnan and provided information for further HIV-1 control and prevention.
\end{abstract}

Keywords: Human immunodeficiency virus-1 (HIV-1), Genetics, Recent infections, Spatial cluster, China

\section{Background}

Since HIV-1 was first identified among intravenous drug users (IDUs) in western Yunnan in 1989, HIV-1 epidemics have dramatically increased in Yunnan [1-3]. Yunnan is considered the HIV-1 gateway in China. Originating in Yunnan, different HIV-1 genotypes have spread to other

\footnotetext{
* Correspondence: jiamanhong@hotmail.com

${ }^{+}$Min Chen and Yanling Ma contributed equally to this work.

Institute for AIDS/STD Control and Prevention, Yunnan Center for Disease Control and Prevention, No. 158, Dongsi Street, Xishan District, Kunming 650022, Yunnan Province, China
}

parts of China $[1,4]$. Over the past nearly 30 years, unprotected sexual contact has replaced intravenous drug injection as the predominant route of HIV-1 transmission [2]. By the end of 2014, the number of people living with HIV/AIDS (PLWHA) in Yunnan was 80,610 (including 50,426 people living with HIV and 30,184 AIDS patients), which was the highest among all provinces in China. Among all PLWHA in Yunnan, 68.9\% received antiretroviral therapy, which was higher than the national average (63.2\%). Among the annual newly reported cases, the proportion of cases attributed to intravenous drug use 
decreased from $28.6 \%$ in 2009 to $9.3 \%$ in 2014; the proportion of heterosexually infected cases increased from $62.5 \%$ in 2009 to $85.1 \%$ in 2014, while the proportion of homosexually infected cases increased from $1.4 \%$ in 2009 to 4.3\% in 2014.

Initially, subtype B and subtype $C$ emerged among IDUs [5-7], and CRF01_AE was found among female sex workers (FSWs) [8]. In the 1990s, CRF07_BC and CRF08_ $\mathrm{BC}$ formed through the recombination of subtype $\mathrm{B}$ and subtype $\mathrm{C}$, which were circulating among IDUs in Yunnan and transmitted along drug trafficking routes $[9,10]$. Through the populations with the dual risk factors of intravenous drug use and heterosexual contact [11], CRF08_BC and CRF07_BC emerged in the heterosexually transmitted population [12]. In the late 2000s, CRF08_BC became the chief HIV-1 genotype among both heterosexuals and IDUs, and the composition of HIV-1 genotypes was nearly the same in these two populations [12]. The first HIV-positive man who had sex with men (MSM) in Yunnan was reported in 2004. The surveillance showed that CRF01_AE and CRF07_BC were the main strains among MSM, accounting for more than $90 \%$ of cases [13]. Recently, CRF55_01B and CRF59_01B were also detected in MSM in Yunnan [14], which were first identified among MSM in southern China and northeastern China, respectively $[15$, 16]. Moreover, the spatial distribution of HIV-1 genotypes dynamically changed. The main HIV-1 genotypes spread from the original areas to other areas of Yunnan. For example, CRF01_AE was transmitted from western to eastern Yunnan, and CRF08_BC and CRF07_BC were transmitted from southeast of Yunnan to other areas.

The genetic diversity of HIV-1 is striking in Yunnan. In addition to the abovementioned subtypes and circulating recombinant forms (CRFs), a multitude of URFs were also detected in Yunnan $[12,17]$. In the late 2000s, the proportion of URFs ranked second only to that of CRF08_BC in recent infections [12]. These URFs contributed to the formation of novel CRFs. In recent years, at least nine CRFs were identified in western Yunnan [18-25]. These CRFs consist of components from subtype $B$, subtype $C$ and CRF01_AE. A respective phylogenetic study showed that some of these CRFs originated in the early 1990s [9].

With the development of HIV-1 epidemics, the demographic profile of those infected has changed, while the characteristics and distribution of HIV-1 genotypes have also changed. To track the changing trend of HIV-1 genotypes in Yunnan, we performed an HIV-1 molecular epidemiological study among a recently infected population in Yunnan in 2015.

\section{Methods}

\section{Study participants and sample collection}

From January 2015 to June 2015, 6357 HIV/AIDS cases were newly reported through different screening methods in Yunnan Province. Before carrying out the recent HIV-1 infection assay, 1755 long-term infected cases with $\mathrm{CD} 4^{+}$ $\mathrm{T}$ lymphocytes $<200$ cells/ $\mu$ l or AIDS-defining illnesses were excluded. The remaining 4602 cases were further tested for the status of recent HIV-1 infection. The adults' written consents were provided by themselves. The juveniles' consents for HIV testing were provided by their guardians, if they had HIV, written consents about this study for HIV control and prevention were further obtained from their guardians when informing testing results. The study was approved by the Biomedical Ethics Review Committee of Yunnan Province.

\section{Recent HIV-1 infection tested with BED-capture enzyme immunoassay (CEIA)}

BED-capture enzyme immunoassay (BED-CEIA) (Calypte Biomedical Corporation, Portland, OR, USA) was used to test for the recency of HIV-1 infection. According to the manufacturer's instructions, plasma specimens were first tested individually. If the normalized OD (ODn) was > 1.2, the specimen was classified as having a long-term infection. Specimens with ODn $<1.2$ were tested again in triplicate for confirmation. In confirmatory testing, specimens with ODn $<0.8$ were classified as recent infections. According to the evaluation carried out in China, the duration of a recent HIV infection determined by BED-CEIA was 168 days after seroconversion.

\section{Amplification of HIV-1 gene fragments}

According to the manufacturer's instructions, viral RNA was extracted from $140 \mu \mathrm{l}$ of plasma using the QIAamp Viral RNA Mini Kit (Qiagen, Valencia, CA, USA). The partial gag gene (HXB2: 781-1861), partial pol gene (HXB2: 2147-3462) and partial env gene (HXB2: $7002-$ 7541) were amplified using nested polymerase chain reactions (PCR). The successfully amplified products were sent to SinoGenoMax Co. (Beijing, China) for sequencing. The primers and procedures for nested PCR and the primers for sequencing were described in a previous study [13].

\section{Sequence analysis}

The sequences were assembled using Sequencher 5.1 (Gene Codes, Ann Arbor, MI). The assembled sequences were aligned with Bio-Edit 7.0 software and further manually edited. HIV-1 reference sequences were selected and downloaded from the HIV databases of the Los Alamos National Laboratory (LANL) (https://www. hiv.lanl.gov). Neighbour-joining phylogenetic trees were construed with the Kimura 2-parameter model with 1000 bootstrap replicates with MEGA version 6.0 [26]. The sequences with possible intersubtype recombination were analysed with the Recombination Identification Programme (RIP, version 3.0; https://www.hiv.lanl.gov). 
HIV-1 genotyping was based on at least two segments of the gag, pol and env genes. Samples with only one gene segment were excluded. Finally, 260 samples had gag, pol and env segments, 108 had gag and pol segments, 45 had gag and env segments, and 26 had pol and env segments. The above 439 samples were considered to have achieved reliable HIV-1 genotyping.

\section{Geographic distribution analysis and spatial scan statistics} analysis of HIV-1 genotypes

The distribution density of each HIV-1 genotype at the county level was displayed using a dot density map, within which the number of dots in a county represented the proportion of cases with a given genotype in this county among all the cases successfully genotyped. One dot represented $0.025 \%$ of the population.

The spatial scanning statistic is used to detect possible spatial clusters by scanning across space with a radius varying circular window. The null hypothesis is that the risks of event occurrence are equal between the inside and outside windows, while the alternative hypothesis is that the risks are different between the inside and outside windows.

Under the Poisson assumption, the log likelihood ratio (LLR) is calculated as:

$$
L L R=\left(\frac{c}{E[c]}\right)^{c}\left(\frac{C-c}{C-E[c]}\right)^{C-c} I()
$$

where $\mathrm{C}$ is the total number of cases; $\mathrm{c}$ is the observed number of cases within the window; $\mathrm{E}[\mathrm{c}]$ is the covariate adjusted expected number of cases within the window under the null-hypothesis; $\mathrm{I}($ ) is an indicator function.

For each location, by varying the size, a window with the maximum likelihood will be found. The $p$-value is obtained through Monte Carlo simulations, by comparing the rank of the maximum likelihood from the real data set with those from random simulated data sets. If this rank is $\mathrm{R}$, then $p=\mathrm{R} /(1+\mathrm{n})$, where $\mathrm{n}$ is the number of random simulations. Among the regions with statistical significance, the regions with the maximum likelihood are defined as the most likely cluster (primary cluster), and the others with a smaller likelihood are defined as secondary clusters.

The relative risk (RR) represents the aggregation risk of the cluster compared with the rest of the regions. RR is defined as the estimated risk within the cluster divided by the estimated risk outside the cluster. The mathematical notation is as follows:

$$
R R=\frac{c / E[c]}{(C-c) /(C-E[c])}
$$

where the meaning of the symbols are the same as above.
In this study, SaTScan 9.6 was used for the spatial scan statistics analysis [27]. A Poisson-based model combined with circular scan windows was used. For the analysis of each HIV-1 genotype, three types of files (case file, population file and coordinates file) were submitted into SaTScan. The case file contained the numbers of cases with a given HIV-1 genotype in each county. The population file contained the number of populations in each county. The coordinates file contained the coordinates of each county. The most commonly used maximum spatial cluster size is $50 \%$ of the population at risk [2834]. To choose the maximum spatial cluster size, a sensitivity analysis was performed with 10,30 and $50 \%$ of the population at risk in the spatial window (Additional file 1: Table S1). The significant clusters of CRF01_AE, CRF07_BC, CRF08_BC and URFs showed no difference when using these settings. Therefore, $10 \%$ of the population at risk was used as the maximum spatial cluster size. The spatial clusters were mapped using Quantum GIS [35].

\section{Genotypic analysis of HIV-1 drug resistance}

Surveillance drug resistance mutations (SDRMs) within the obtained pol sequences were screened using the Calibrated Population Resistance (CPR) Tool (Version 6.0) on Stanford HIV Drug Resistance Database (http:// hivdb.stanford.edu) [36].

\section{Statistical analysis}

Statistical analyses were performed with the SPSS 21.0 statistical analysis software package (SPSS Inc. Chicago, IL). Demographic characteristics associated with HIV-1 genotypes were first analysed with univariate logistic regression. For each HIV-1 genotype, variables marginally significant with $p<0.10$ in univariate logistic analysis were further analysed with multivariate logistic regression. The trend chi-square test was performed with the linear-by-linear association method under the chi-square test of SPSS. All tests were two-tailed and statistical significance was considered when $p$-value $<0.05$.

\section{Results}

\section{Demographic characteristics of the study participants}

As shown in Fig. 1, during the first half of 2015, 6,357 HIV/AIDS cases were newly diagnosed through the different screening methods in Yunnan Province. Based on information regarding disease progression and a BEDCEIA assay, 586 samples were identified as recent infections and were subjected to HIV-1 gene amplification. Finally, $444 \mathrm{gag}, 411 \mathrm{pol}$ and $357 \mathrm{env}$ sequences were obtained, which were further subjected to phylogenetic analyses (Additional file 2: Figure S1, Additional file 3: Figure S2, Additional file 4: Figure S3). By combining the genotyping of at least two gene segments, 439 


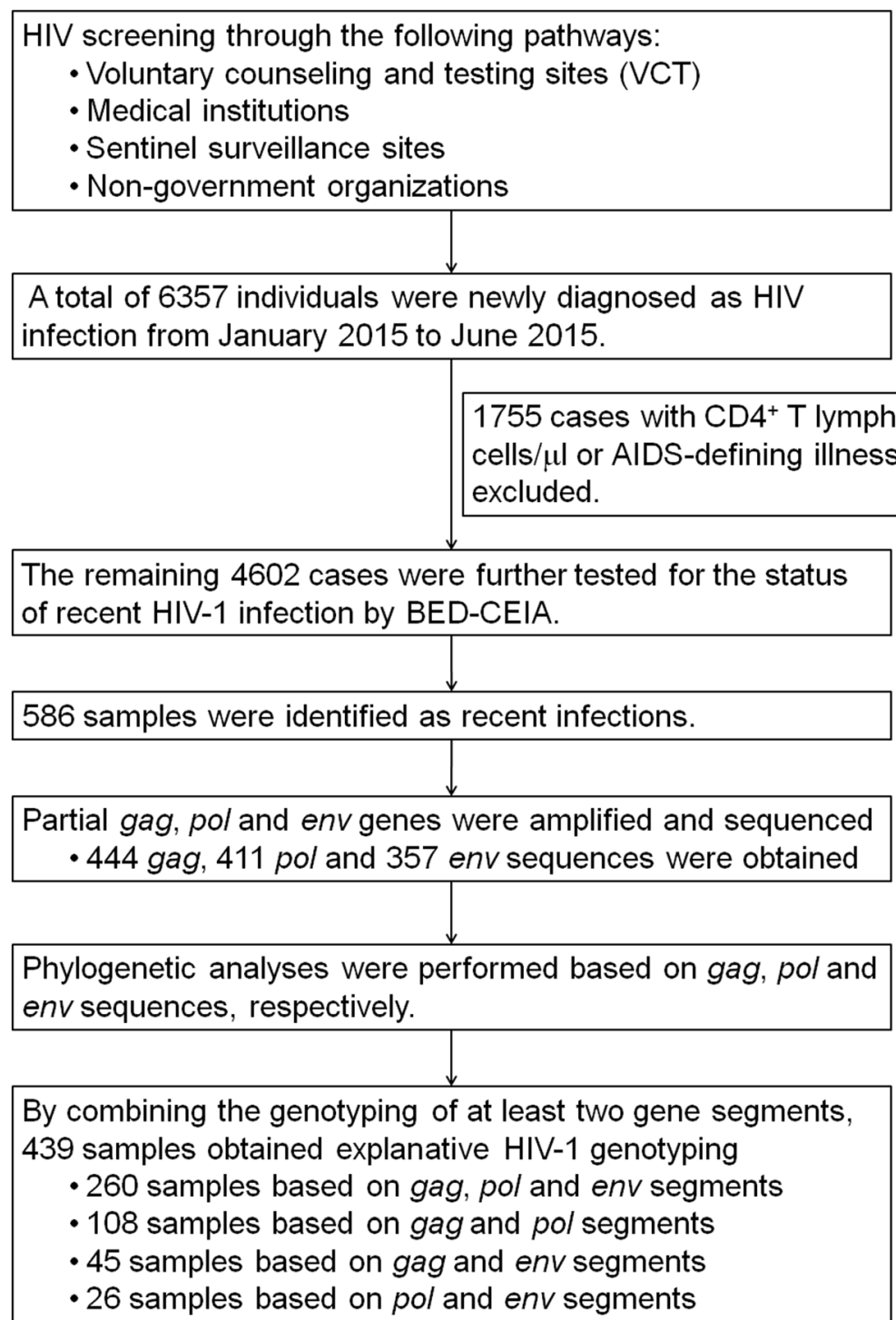

Fig. 1 Flow chart of the study process. The flow chart shows the overall study process, including HIV-1 screening and confirmation, recent infection testing and HIV-1 genotyping

samples obtained explanative HIV-1 genotyping. The constituent of the 439 participants showed no significant differences with that of the total 586 recently infected individuals (Additional file 5: Table S2).

Of the 439 participants, $62.0 \%(272 / 439)$ were males, and $38.0 \%(167 / 439)$ were females; the median age was 35 years (range: 15-81 years); Han ethnicity accounted for $62.0 \%$ (272/439), while other ethnicities accounted for $38.0 \%$, including Yi, Hani, Dai, Zhuang, Jingpo, Bai, Hui, Wa, Lahu, Lisu, Miao, Yao, Man, Achang, Buyi, Naxi and Nu. Regarding marital status, 33.7\% (148/439) were single, $43.7 \%(192 / 439)$ were married, and $22.6 \%$ (99/439) were divorced or widowed. Of the 439 participants, $5.0 \%$ (22/439) were non-Chinese, most of which were Burmese living Dehong Prefecture (72.7\%, 16/22).
For the transmission routes, heterosexual contact, homosexual contact and intravenous drug injection accounted for $81.1 \%(356 / 439), 11.2 \%$ (49/439) and $7.7 \%$ (34/439), respectively.

HIV-1 genotypes in recent HIV infections

Among the 439 samples successfully genotyped, more than ten HIV-1 genotypes were identified, including two subtypes, nine CRFs and six discrete URFs. CRF08_BC was the most common (45.3\%, 199/439), followed by CRF07_BC (19.4\%, 85/439), URFs (18.2\%, 80/439), CRF01_AE (11.4\%, 50/439), subtype C (2.1\%, 9/439), CRF85_BC (1.1\%, 5/439), CRF55_01B (0.9\%, 4/439), subtype B (0.5\%, 2/439), CRF64 BC (0.5\%, 2/439), CRF59_01B (0.2\%, 1/439), CRF83_cpx $(0.2 \%, 1 / 439)$ and CRF87_cpx $(0.2 \%, 1 / 439)$. Among the six 
discrete URFs, $\mathrm{BC}$ recombinants were the predominant recombinant form $(66.3 \%, 53 / 80)$, followed by BC/CRF01_AE (10.0\%, 8/80), CRF07_BC/CRF01_AE (8.8\%, 7/80), B/ CRF01_AE (6.3\%, 5/80), C/CRF01_AE $(5.0 \%, 4 / 80)$ and CRF07_BC/CRF08_BC $(3.8 \%, 3 / 80)$.

\section{Demographic characteristics associated with HIV-1 genotypes}

Demographic characteristics associated with HIV-1 genotypes were further analysed using logistic regression (Table 1). The results of the multivariate analyses showed that homosexual contact was significantly associated with CRF01_AE and CRF07_BC infection; females, Chinese, heterosexual contact and intravenous drug injection were significantly associated with CRF08_ $\mathrm{BC}$ infection; males and non-Chinese had a higher risk of URF infection than females.

\section{Spatial distribution of HIV-1 genotypes}

The spatial distribution of HIV-1 genotypes in recent infections was analysed at the county level (Fig. 2). CRF08_ $\mathrm{BC}$ was the most widely distributed genotype, which was found in 54.3\% (70/129) of the counties in Yunnan Province. However, more CRF08_BC cases were found in the six eastern prefectures (Zhaotong, Qujing, Kunming, Yuxi, Honghe and Wenshan). The other three main genotypes, CRF07_BC, URFs and CRF01_AE, were found in 38.0\% (49/129), 34.1\% (44/129) and 20.2\% (26/129) of all counties, respectively. The distributions of subtype C, CRF85 BC, CRF55_01B, subtype B, CRF64_BC, CRF59_01B, CRF83_cpx and CRF87_cpx were limited.

Spatial clusters were further analysed using spatial scan statistics (Fig. 3). For CRF08_BC, two non-overlapping statistically significant clusters were detected. Among them, the most likely cluster $(R R=4.78)$ included 11 counties in Honghe Prefecture and Wenshan Prefecture, and the secondary clusters $(R R=4.24)$ included two counties in Zhaotong Prefecture. In these two spatial clusters, 95.2 and $90.0 \%$ of observed cases were infected through heterosexual contact (Table 2). For CRF07_BC, there was only one most likely cluster $(\mathrm{RR}=3.68)$ detected in Kunming Prefecture (Table 2). For CRF01_AE, two non-overlapping statistically significant clusters were detected. Among them, the most likely cluster $(\mathrm{RR}=17.41)$ was located in one county in Dali Prefecture, and the secondary cluster $(R R=5.36)$ included six counties in Kunming Prefecture and Yuxi Prefecture. In the most likely cluster and the secondary cluster, 80.0 and $63.6 \%$ of observed cases were infected through homosexual contact (Table 2). For URFs, two non-overlapping statistically significant clusters were detected. Among them, the most likely cluster $(R R=9.62)$ was located in the west, including six counties in Dehong Prefecture and Baoshan Prefecture, in which
$60.9 \%$ of observed cases were infected through intravenous drug use; the secondary cluster $(\mathrm{RR}=3.51)$ was located in the southeast and included nine counties in Honghe Prefecture and Wenshan Prefecture, in which $100.0 \%$ of observed cases were infected through heterosexual contact (Table 2).

\section{Genotypic analysis of transmitted drug resistance (TDR)}

Among the 411 participants with pol sequences, ten (2.4\%) were identified as harbouring SDRMs. As shown in Table 3, 0.5\% (2/411), $1.2 \%(5 / 411)$, and $0.7 \%(3 / 411)$ of the sequences harboured SDRMs to nucleoside reverse transcriptase inhibitors (NRTIs), non-nucleoside reverse transcriptase inhibitors (NNRTIs), and protease inhibitors (PIs), respectively. As shown in Table 4, the prevalence of SDRMs increased with age (trend $X^{2}=$ 4.739, $p=0.033)$.

\section{Discussion}

In this study, we performed a cross-sectional HIV-1 molecular epidemiological study to track the characteristics and distribution of HIV-1 genotypes in recent infections in Yunnan. For the first time, the spatial epidemiological characteristics of HIV-1 genotypes among recent infections were analysed.

As described in the previous studies [12], CRF08_BC, CRF07_BC, URFs and CRF01_AE were the main HIV-1 genotypes circulating in Yunnan. However, compared with the surveillance carried out in 2009, the ranks of these four genotypes changed. The proportion of CRF08_BC among recent infections remained the highest and showed no significant difference from 2009 to 2015 (40.8\% in $2009,47.0 \%$ in 2014 and $45.3 \%$ in 2015 ; $\left.X^{2}=1.605, p=0.450\right)[12,37]$. The proportion of CRF07_ $\mathrm{BC}$ increased from fourth to second place and showed a statistically increasing trend for 2009-2015 (9.2\% in $2009,14.4 \%$ in 2014 and $18.3 \%$ in 2015 ; trend $\chi^{2}=8.557$, $p=0.003)$. The proportion of URFs declined from second to third place and showed a decreasing trend $(27.7 \%$ in $2009,18.2 \%$ in 2014 and $18.2 \%$ in $2015 ; \chi^{2}=6.272$, $p=0.045)$. The proportion of CRF01_AE declined from third to fourth place and showed a decreasing trend that was not statistically significant $(18.5 \%$ in $2009,15.8 \%$ in 2014 and $11.4 \%$ in $\left.2015 ; \chi^{2}=5.437, p=0.064\right)$. The overall increase in CRF07_BC may be partly due to the increase in CRF07_BC among homosexual contact. Among MSM, the proportion of CRF07_BC increased from $17.2 \%$ in 2014 to $42.9 \%$ in $2015\left(\chi^{2}=5.379, p=\right.$ $0.026)$.

In addition to the above four predominant genotypes, two subtypes and six CRFs were found in recent infections. The genetic diversity of HIV-1 constitutes a characteristic of the HIV-1 epidemic in Yunnan. HIV-1 recombination was active in the Yunnan-Myanmar 
Table 1 Demographic characteristics associated with HIV-1 genotypes

\begin{tabular}{|c|c|c|c|c|c|c|}
\hline & \multirow[t]{2}{*}{ Total } & \multirow{2}{*}{$\begin{array}{l}\text { Subjects with given } \\
\text { HIV-1 genotype }\end{array}$} & \multicolumn{2}{|c|}{$\underline{\text { Univariate analysis }}$} & \multicolumn{2}{|c|}{ Multivariate analysis } \\
\hline & & & $p$ & OR $(95 \% \mathrm{Cl})$ & $p$ & OR $(95 \% \mathrm{Cl})$ \\
\hline \multicolumn{7}{|l|}{ CRF01_AE } \\
\hline \multicolumn{7}{|l|}{ Gender } \\
\hline Female & 167 & 12 & - & 1.000 & - & 1.000 \\
\hline Male & 272 & 38 & 0.033 & $2.098(1.063 \sim 4.140)$ & 0.431 & $1.356(0.636 \sim 2.889)$ \\
\hline Age & & & 0.256 & & & \\
\hline$\leq 30$ & 174 & 25 & - & 1.000 & & \\
\hline $31-50$ & 182 & 16 & 0.102 & $0.574(0.295 \sim 1.117)$ & & \\
\hline$\geq 51$ & 83 & 9 & 0.437 & $0.725(0.322 \sim 1.632)$ & & \\
\hline \multicolumn{7}{|l|}{ Nationality } \\
\hline Chinese & 417 & 49 & - & 1.000 & & \\
\hline Non-Chinese & 22 & 1 & 0.320 & $0.358(0.047 \sim 2.718)$ & & \\
\hline \multicolumn{7}{|l|}{ Race/ethnicity } \\
\hline Han & 272 & 31 & - & 1.000 & & \\
\hline Others & 167 & 19 & 0.995 & $0.998(0.544 \sim 1.831)$ & & \\
\hline Marital Status & & & 0.034 & & 0.706 & \\
\hline Married & 192 & 15 & - & 1.000 & - & 1.000 \\
\hline Divorced/Widowed & 99 & 10 & 0.510 & $1.326(0.573 \sim 3.070)$ & 0.727 & $1.166(0.493 \sim 2.754)$ \\
\hline Unmarried & 148 & 25 & 0.012 & 2.398 (1.215 (4.735) & 0.404 & $1.392(0.640 \sim 3.026)$ \\
\hline Infection Routes & & & $<0.001$ & & $0.001^{*}$ & \\
\hline Heterosexual contact & 354 & 32 & - & 1.000 & - & 1.000 \\
\hline Homosexual contact & 49 & 18 & $<0.001$ & $5.843(2.945 \sim 11.591)$ & $<0.001^{*}$ & 4.469 (2.016 9.908) \\
\hline Intravenous drug injection & 36 & 0 & 0.998 & - & 0.998 & - \\
\hline \multicolumn{7}{|l|}{ CRF07_BC } \\
\hline \multicolumn{7}{|l|}{ Gender } \\
\hline Male & 272 & 55 & - & 1.000 & & \\
\hline Female & 167 & 30 & 0.562 & $0.864(0.527 \sim 1.415)$ & & \\
\hline Age & & & 0.004 & & 0.165 & \\
\hline$\geq 51$ & 83 & 10 & - & 1.000 & - & 1.000 \\
\hline $31-50$ & 182 & 28 & 0.473 & 1.327 (0.612 2.878) & 0.516 & $1.305(0.585 \sim 2.908)$ \\
\hline$\leq 30$ & 174 & 47 & 0.009 & $2.702(1.288 \sim 5.667)$ & 0.092 & $2.136(0.884 \sim 5.158)$ \\
\hline \multicolumn{7}{|l|}{ Nationality } \\
\hline Chinese & 417 & 81 & - & 1.000 & & \\
\hline Non-Chinese & 22 & 1 & 0.106 & $0.189(0.025 \sim 1.423)$ & & \\
\hline \multicolumn{7}{|l|}{ Race/ethnicity } \\
\hline Han & 272 & 60 & - & 1.000 & - & 1.000 \\
\hline Others & 167 & 25 & 0.070 & $0.622(0.373 \sim 1.039)$ & 0.098 & $0.630(0.364 \sim 1.089)$ \\
\hline Marital Status & & & 0.003 & & 0.820 & \\
\hline Divorced/Widowed & 99 & 14 & - & 1.000 & - & 1.000 \\
\hline Married & 192 & 29 & 0.826 & $1.080(0.542 \sim 2.153)$ & 0.810 & $1.090(0.538 \sim 2.210)$ \\
\hline Unmarried & 148 & 42 & 0.010 & $2.406(1.232 \sim 4.696)$ & 0.545 & $1.283(0.573 \sim 2.871)$ \\
\hline Infection Routes & & & $<0.001$ & & $0.014^{*}$ & \\
\hline Heterosexual contact & 354 & 58 & - & 1.000 & - & 1.000 \\
\hline Intravenous drug injection & 36 & 6 & 0.965 & 1.021 (0.407 2.563) & 0.960 & 1.025 (0.397 2.642) \\
\hline
\end{tabular}


Table 1 Demographic characteristics associated with HIV-1 genotypes (Continued)

\begin{tabular}{|c|c|c|c|c|c|c|}
\hline \multirow{3}{*}{ Homosexual contact } & \multirow{3}{*}{$\begin{array}{l}\text { Total } \\
49\end{array}$} & \multirow{3}{*}{$\begin{array}{l}\text { Subjects with given } \\
\text { HIV-1 genotype } \\
21\end{array}$} & \multicolumn{2}{|c|}{ Univariate analysis } & \multicolumn{2}{|c|}{$\underline{\text { Multivariate analysis }}$} \\
\hline & & & $p$ & OR $(95 \% \mathrm{Cl})$ & $p$ & OR $(95 \% \mathrm{Cl})$ \\
\hline & & & $<0.001$ & $3.828(2.035 \sim 7.201)$ & $0.004^{*}$ & $2.767(1.392 \sim 5.501)$ \\
\hline \multicolumn{7}{|l|}{ CRF08_BC } \\
\hline \multicolumn{7}{|l|}{ Gender } \\
\hline Male & 272 & 103 & - & 1.000 & - & 1.000 \\
\hline Female & 167 & 96 & $<0.001$ & 2.219 (1.498 3.285) & $0.012^{*}$ & 1.760 (1.132 2.738) \\
\hline Age & & & 0.001 & & 0.216 & \\
\hline$\leq 30$ & 174 & 60 & - & 1.000 & - & 1.000 \\
\hline $31-50$ & 182 & 93 & 0.002 & $1.985(1.296 \sim 3.042)$ & 0.118 & $1.512(0.900 \sim 2.540)$ \\
\hline$\geq 51$ & 83 & 46 & 0.002 & 2.362 (1.385 4.029) & 0.121 & 1.696 (0.870 3.308) \\
\hline \multicolumn{7}{|l|}{ Nationality } \\
\hline Non-Chinese & 22 & 1 & - & 1.000 & - & 1.000 \\
\hline Chinese & 417 & 198 & 0.004 & 18.986 (2.531 142.450) & $0.004^{*}$ & 21.061 (2.657 166.944) \\
\hline \multicolumn{7}{|l|}{ Race/ethnicity } \\
\hline Han & 272 & 126 & - & 1.000 & & \\
\hline Others & 167 & 73 & 0.594 & $0.900(0.611 \sim 1.326)$ & & \\
\hline Marital Status & & & $<0.001$ & & 0.807 & \\
\hline Unmarried & 148 & 47 & - & 1.000 & - & 1.000 \\
\hline Married & 192 & 99 & $<0.001$ & $2.288(1.463 \sim 3.577)$ & 0.809 & $1.071(0.614 \sim 1.869)$ \\
\hline Divorced/Widowed & 99 & 53 & 0.001 & $2.476(1.464 \sim 4.186)$ & 0.535 & $1.229(0.641 \sim 2.356)$ \\
\hline Infection Routes & & & $<0.001$ & & $<0.001^{*}$ & \\
\hline Homosexual contact & 49 & 3 & - & 1.000 & - & 1.000 \\
\hline Intravenous drug injection & 36 & 14 & 0.001 & 9.7548 (2.538 37.507) & $<0.001^{*}$ & $14.443(3.452 \sim 60.437)$ \\
\hline Heterosexual contact & 354 & 182 & $<0.001$ & 16.225 (4.954 53.138) & $<0.001^{*}$ & $11.029(3.250 \sim 37.429)$ \\
\hline \multicolumn{7}{|l|}{ URFs } \\
\hline \multicolumn{7}{|l|}{ Gender } \\
\hline Female & 167 & 23 & - & 1.000 & - & 1.000 \\
\hline Male & 272 & 57 & 0.060 & 1.660 (0.979 2.815) & $0.035^{*}$ & $1.853(1.043 \sim 3.292)$ \\
\hline \multicolumn{7}{|l|}{ Age } \\
\hline$\leq 30$ & 174 & 33 & - & 1.000 & & \\
\hline $31-50$ & 182 & 33 & 0.840 & $0.946(0.554 \sim 1.615)$ & & \\
\hline$\geq 51$ & 83 & 14 & 0.684 & $0.867(0.436 \sim 1.725)$ & & \\
\hline \multicolumn{7}{|l|}{ Nationality } \\
\hline Chinese & 417 & 64 & - & 1.000 & - & 1.000 \\
\hline Non-Chinese & 22 & 16 & $<0.001$ & 14.708 (5.546 39.007) & $<0.001^{*}$ & 17.719 (5.624 55.829) \\
\hline \multicolumn{7}{|l|}{ Race/ethnicity } \\
\hline Han & 272 & 42 & - & 1.000 & & \\
\hline Others & 167 & 38 & 0.055 & $1.613(0.989 \sim 2.630)$ & & \\
\hline Marital Status & & & 0.601 & & & \\
\hline Unmarried & 148 & 25 & - & 1.000 & & \\
\hline Married & 192 & 29 & 0.424 & $1.254(0.720 \sim 2.186)$ & & \\
\hline Divorced/Widowed & 99 & 16 & 0.880 & $0.948(0.477 \sim 1.884)$ & & \\
\hline Infection Routes & & & 0.063 & & 0.189 & \\
\hline Homosexual contact & 49 & 5 & - & 1.000 & - & 1.000 \\
\hline
\end{tabular}


Table 1 Demographic characteristics associated with HIV-1 genotypes (Continued)

\begin{tabular}{|c|c|c|c|c|c|c|}
\hline & \multirow[t]{2}{*}{ Total } & \multirow{2}{*}{$\begin{array}{l}\text { Subjects with given } \\
\text { HIV-1 genotype }\end{array}$} & \multicolumn{2}{|c|}{ Univariate analysis } & \multicolumn{2}{|c|}{ Multivariate analysis } \\
\hline & & & $p$ & OR $(95 \% \mathrm{Cl})$ & $p$ & OR $(95 \% \mathrm{Cl})$ \\
\hline Heterosexual contact & 354 & 64 & 0.177 & $1.942(0.741 \sim 5.091)$ & 0.117 & $2.208(0.821 \sim 5.936)$ \\
\hline Intravenous drug injection & 36 & 11 & 0.023 & 3.872 (1.207 12.420) & 0.783 & $1.221(0.296 \sim 5.038)$ \\
\hline
\end{tabular}

*: statistical significance

border area, where URFs were highly prevalent $[9,38]$ and more than ten CRFs were identified recently, including CRF57_BC, CRF62_BC, CRF64_BC, CRF65_ cpx, CRF78_cpx, CRF82_cpx, CRF83_cpx, CRF86_BC, CRF87_cpx, CRF88_BC and CRF96_cpx $[18-25,39]$. In this study, CRF64_BC, CRF83_cpx and CRF87_cpx were found in western Yunnan, which suggested that they were circulating locally. CRF55_01B and CRF59_ 01B were originally identified in MSM outside Yunnan $[15,16]$; CRF85_BC was originally identified in the neighbouring province, Sichuan Province [40]. In this study, these three CRFs were found in eastern Yunnan, where the floating population was relatively larger.

The demographic characteristics associated with HIV1 genotypes were further analysed using multivariate logistic regression. Because heterosexual contact was the major transmission route (81.1\%), the absolute numbers of the main genotypes in this transmission route were the largest. However, the proportions of CRF01_AE and CRF07_BC in homosexual contact were higher than those in heterosexual contact and intravenous drug

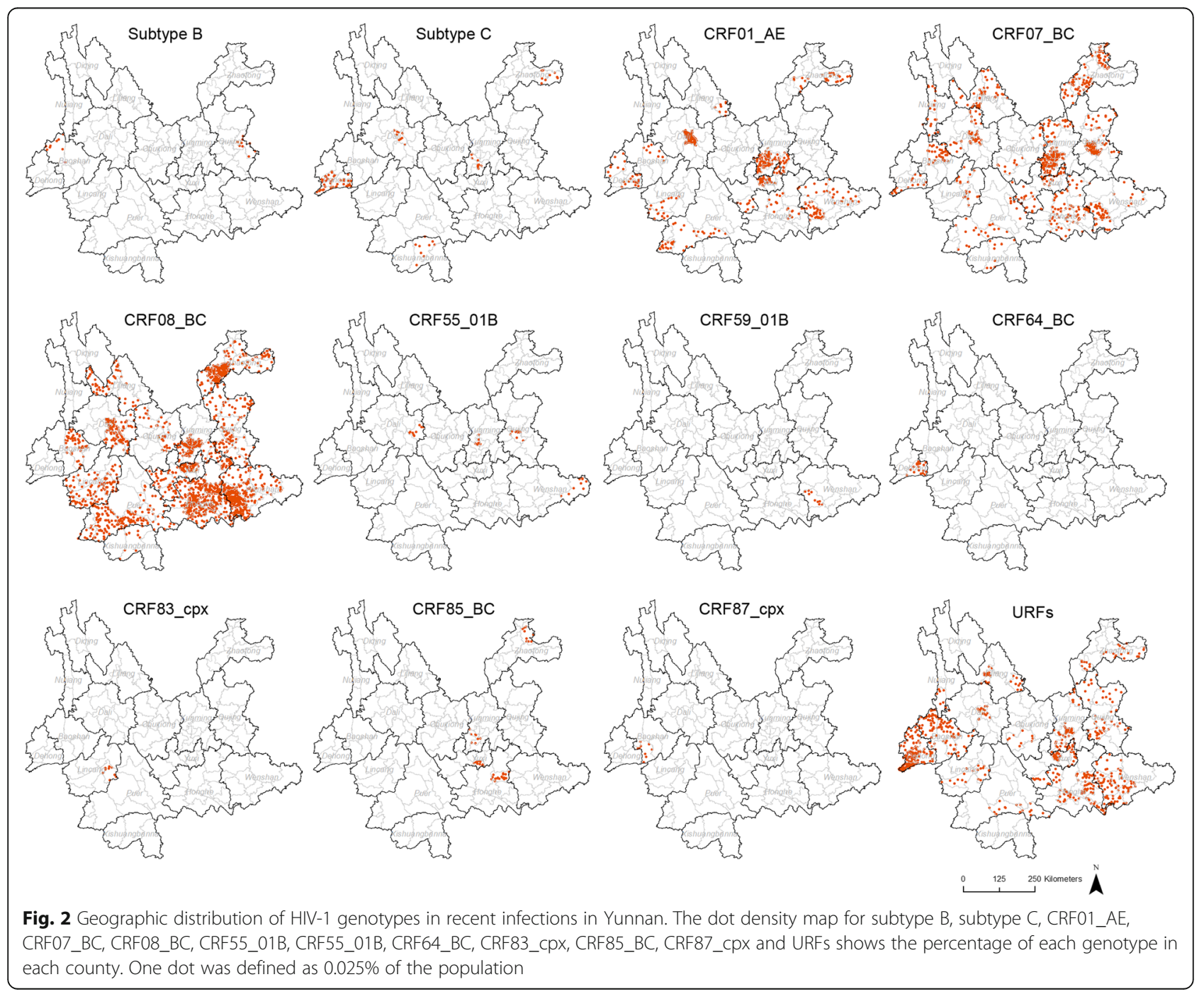




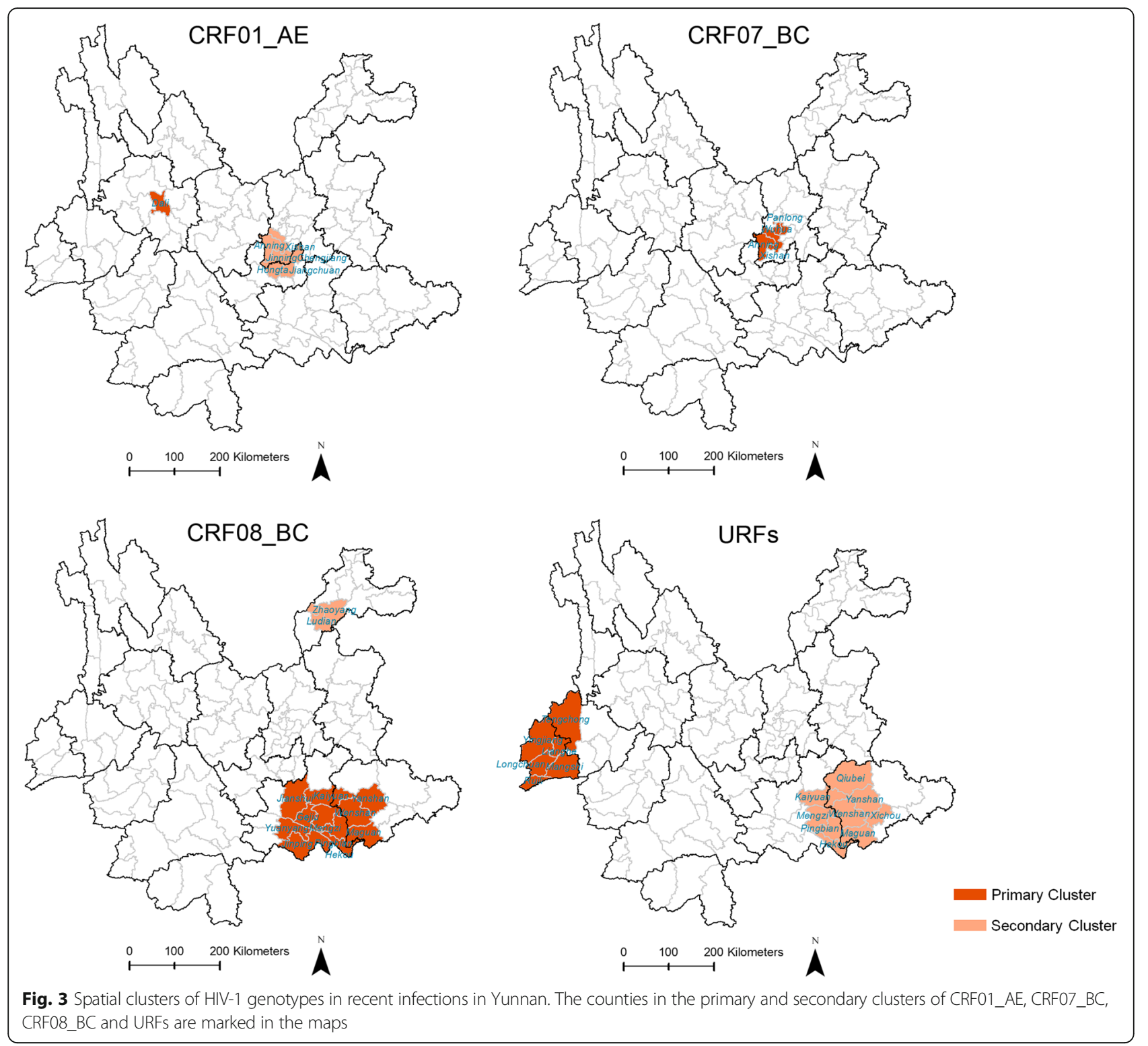

injection, while the proportion of CRF08_BC in homosexual contact was lower than those in the other two transmission routes. These findings suggested that the homosexually transmitted population remained relatively unique from the other two populations. The proportion of CRF08_BC was higher in females. The reason for this finding could be that the predominant transmission route among the female cases was heterosexual contact $(95.8 \%, 160 / 167)$, and in this transmission route, CRF08_ $\mathrm{BC}$ was the predominant HIV-1 genotype. Furthermore, combined with gender and transmission routes, the subjects were divided into five subgroups. The proportion of CRF08_BC was the highest in the subgroup of female heterosexual contact and the lowest in the subgroup of male homosexual contact (Additional file 6: Table S3). We also found that the proportion of URFs among males was higher than that among females, that the proportion of URFs among non-Chinese was higher than that among Chinese. Usually, URFs were identified in dually or multiply infected individuals. This finding suggested that males and non-Chinese tended to have more complex risk factors. To fully understand HIV-1 genetic characteristics among non-Chinese, the further study should be carried out in the border areas.

For the first time, we described the geographic distribution of HIV-1 genotypes among recent infections at the county level, which was more elaborate than the previous study at the prefecture level [12]. Among the four predominant genotypes, the coverage of CRF08_BC was greater than those of CRF07_BC, URFs and CRF01_AE. However, the other genotypes were only found in one to six counties. In some areas, the four predominant 
Table 2 The characteristics of spatial clusters for CRF01_AE, CRF07_BC, CRF08_BC and URFs

\begin{tabular}{|c|c|c|c|c|c|c|c|c|}
\hline \multirow{2}{*}{$\begin{array}{l}\text { HIV-1 } \\
\text { genotypes }\end{array}$} & \multirow[t]{2}{*}{ Counties } & \multirow{2}{*}{$\begin{array}{l}\text { Relative } \\
\text { risk }\end{array}$} & \multirow{2}{*}{$\begin{array}{l}\text { Log likelihood } \\
\text { ratio }\end{array}$} & \multirow{2}{*}{$p$-value } & \multicolumn{4}{|c|}{ Observed cases } \\
\hline & & & & & Total & $\begin{array}{l}\text { Heterosexual } \\
\text { contact }\end{array}$ & $\begin{array}{l}\text { Homosexual } \\
\text { contact }\end{array}$ & $\begin{array}{l}\text { Intravenous } \\
\text { drug injection }\end{array}$ \\
\hline \multicolumn{9}{|l|}{ CRF01_AE } \\
\hline $\begin{array}{l}\text { Primary } \\
\text { Cluster }\end{array}$ & Dali & 17.41 & 18.1 & $6.4 \mathrm{E}-07$ & 10 & $2(20.0 \%)$ & $8(80.0 \%)$ & 0 \\
\hline $\begin{array}{l}\text { Secondary } \\
\text { Cluster }\end{array}$ & $\begin{array}{l}\text { Xishan, Jinning, Anning, Hongta, } \\
\text { Jiangchuan, Chengjiang }\end{array}$ & 5.36 & 8.6 & 7.0E-03 & 11 & $4(36.4 \%)$ & $7(63.6 \%)$ & 0 \\
\hline \multicolumn{9}{|l|}{ CRF07_BC } \\
\hline $\begin{array}{l}\text { Primary } \\
\text { Cluster }\end{array}$ & Wuhua, Panlong, Xishang, Anning & 3.68 & 8.3 & $1.4 \mathrm{E}-02$ & 16 & $7(43.8 \%)$ & $7(43.8 \%)$ & $2(12.5 \%)$ \\
\hline \multicolumn{9}{|l|}{ CRF08_BC } \\
\hline $\begin{array}{l}\text { Primary } \\
\text { Cluster }\end{array}$ & $\begin{array}{l}\text { Gejiu, Kaiyuan, Mengzi, Pingjian, } \\
\text { Jianshui, Yuanyang, Jinping, Hekou, Wenshan, } \\
\text { Yanshan, Maguan }\end{array}$ & 4.78 & 41.2 & 4.4E-16 & 63 & $60(95.2 \%)$ & $1(1.6 \%)$ & $2(3.2 \%)$ \\
\hline $\begin{array}{l}\text { Secondary } \\
\text { Cluster }\end{array}$ & Zhaoyang, Ludian & 4.24 & 13.0 & $1.5 \mathrm{E}-04$ & 20 & $18(90.0 \%)$ & 0 & $2(10.0 \%)$ \\
\hline \multicolumn{9}{|l|}{ URFs } \\
\hline $\begin{array}{l}\text { Primary } \\
\text { Cluster }\end{array}$ & $\begin{array}{l}\text { Tenchong, Ruili, Mangshi, Lianghe, Yingjiang, } \\
\text { Longchuan }\end{array}$ & 9.62 & 28.2 & $4.1 \mathrm{E}-11$ & 23 & $14(60.9 \%)$ & 0 & $9(39.1 \%)$ \\
\hline $\begin{array}{l}\text { Secondary } \\
\text { Cluster }\end{array}$ & $\begin{array}{l}\text { Kaiyuan, Mengzi, Pingbian, Hekou, Wenshan, } \\
\text { Yanshan, Xichou, Maguan, Qiubei }\end{array}$ & 3.51 & 7.7 & $2.4 \mathrm{E}-02$ & 16 & $16(100.0 \%)$ & 0 & 0 \\
\hline
\end{tabular}

genotypes displayed a clustering tendency. To reveal the spatial clustering characteristics, spatial scanning analysis was performed. There were two statistically significant spatial clusters for CRF08_BC, among which the most likely cluster was located in southeast Yunnan Province and bordered with Vietnam. Strikingly, more than $90 \%$ of observed cases in these two spatial clusters were infected through heterosexual contact, which suggested that more efforts for the prevention of heterosexual contact transmission should be taken in these areas.

Among the two statistically significant clusters for CRF01_AE, the most likely cluster in Dali Prefecture and the second secondary cluster in Kunming and Yuxi Prefecture were largely composed of the cases infected through homosexual contact. Among MSM identified as recent HIV infections in this study, the proportions of MSM identified in Kunming and Dali were $46.8 \%(24 / 59)$ and $16.9 \%(11 / 59)$, respectively, and ranked the top two (Additional file 7: Table S4). If the HIV incidence is assumed to be roughly the same among MSM, the population sizes of MSM in Kunming and Dali were the highest in Yunnan Province. Strikingly, the RR of the most likely cluster for CRF01_AE was the highest among all the detected spatial clusters. RR means how many times the estimated risk in cluster is as high as the estimated risk outside the cluster. The higher RR reflects the higher aggregation degree of the specific risk. Thus, this

Table 3 Demographic characteristics of ten individuals harbouring transmitted drug resistance associated mutations

\begin{tabular}{|c|c|c|c|c|c|c|c|c|}
\hline \multirow{2}{*}{$\begin{array}{l}\text { Sequence } \\
\text { ID }\end{array}$} & \multirow[t]{2}{*}{ Prefecture } & \multirow[t]{2}{*}{ Age } & \multirow[t]{2}{*}{ Marriage status } & \multirow[t]{2}{*}{ Infection route } & \multirow[t]{2}{*}{ Genotype } & \multicolumn{3}{|c|}{ Drug resistance associated mutations } \\
\hline & & & & & & NRTI & NNRTI & $\mathrm{Pl}$ \\
\hline 15R057 & Wenshan & 47 & Unmarried & Heterosexual contact & CRF08_BC & T69D & None & None \\
\hline 15R073 & Wenshan & 30 & Married & Heterosexual contact & $\mathrm{BC}$ & None & $\mathrm{K} 103 \mathrm{~N}, \mathrm{P} 225 \mathrm{H}$ & None \\
\hline 15R089 & Wenshan & 44 & Unmarried & Heterosexual contact & $\mathrm{BC}$ & None & K103 N & None \\
\hline 15R091 & Kunming & 65 & Divorced/widowed & Heterosexual contact & CRF07_BC & None & None & F53Y \\
\hline 15R099 & Kunming & 55 & Married & Heterosexual contact & CRF08_BC & K70E & None & None \\
\hline 15R289 & Qujing & 75 & Divorced/widowed & Heterosexual contact & CRF08_BC & None & K103 N & None \\
\hline 15R366 & Dali & 38 & Married & Heterosexual contact & C & None & $\mathrm{K} 101 \mathrm{E}$ & None \\
\hline 15R377 & Honghe & 81 & Divorced/widowed & Heterosexual contact & CRF01_AE & None & None & M46I \\
\hline 15R421 & Honghe & 41 & Married & Heterosexual contact & CRF08_BC & None & None & G73S \\
\hline 15DHR38 & Dehong & 41 & Married & Intravenous drug injection & CRF01_AE/BC & None & G190A & None \\
\hline
\end{tabular}


Table 4 The prevalence of SDRMs in different age groups

\begin{tabular}{lllcl}
\hline Age & Total & Subjects with SDRMs & Trend $x 2$ & $p$ \\
\hline$<30$ & 168 & $1(0.6 \%)$ & 4.739 & 0.033 \\
$31-50$ & 165 & $5(3.0 \%)$ & & \\
$\geq 51$ & 78 & $4(5.1 \%)$ & & \\
\hline
\end{tabular}

suggested that the risk of CRF01_AE infection displayed a high clustering tendency in this cluster.

As mentioned above, Dehong Prefecture, one of the prefectures bordering Myanmar, was a hot spot of active HIV-1 genetic recombination. In this study, the most likely cluster for URFs was detected in the ChinaMyanmar border area, including four counties in Dehong Prefecture and one county in Baoshan Prefecture. In this URF cluster, $65.2 \%$ (15/23) of the observed cases were Burmese residing in Dehong and Baoshan, and the transmission risks of heterosexual contact $(60.9 \%, 14 / 23)$ and intravenous drug use $(39.1 \%, 9 / 23)$ coexist. Recent studies also suggested that Burmese living in Yunnan contributed to cross-border transmission of HIV-1 in the China-Myanmar border areas [41, 42]. These results suggested that AIDS prevention and control in border areas should be further considered. The secondary cluster for URFs was located in the nine counties in Honghe Prefecture and Wenshan Prefecture, which almost overlapped with the most likely cluster for CRF08_BC. In this URF cluster, 100\% of the observed cases were infected through heterosexual contact, which further suggested the high transmission risk of heterosexual contact in this area. Overall, the spatial clustering analysis for recent infections could provide clues for potential transmission risk, based on which the targeted measures for AIDS control and prevention should be developed.

The prevalence of SDRMs in recent infections was $2.4 \%$ in 2015 , which was not significantly higher than that in $2014\left(1.8 \%, X^{2}=0.263, p=0.791\right)$ [37]. However, we found that the prevalence of TDR increased with age. In the group that was above 50 years of age, the prevalence of TDR was over 5\% and reached a moderate level, which should be considered. To control HIV-1 TDR, a priority is to strengthen the standard management of patients and regularly perform drug resistance surveillance.

\section{Conclusions}

With the occurrence of HIV-1 epidemics in Yunnan, HIV-1 genotypes have become more diverse. Our study revealed two subtypes, nine CRFs and six discrete URFs circulating in recent infections, among which CRF08 BC, CRF07_BC, URFs, and CRF01_AE were still the predominant strains. The distribution of the four predominant genotypes in the population was associated with demographic characteristics. The geographic distribution of HIV-1 genotypes in recent infections was further analysed at the county level. The four predominant genotypes demonstrated a clustering tendency in some geographic areas, which was confirmed by the spatial scan statistics. Some spatial clusters were associated with a specific transmission route. TDR-associated mutations remained low in recent infections. Our findings provide valuable information to improve strategies to prevent new infections.

\section{Additional files}

Additional file 1: Table S1. The comparison of scan statistical results by using different percentages of the population at risk. (PDF $70 \mathrm{~kb}$ )

Additional file 2: Figure S1. Neighbour-joining phylogenetic tree of the partial gag gene. The scale bar indicates $5 \%$ nucleotide sequence divergence. Values on the branches represent the percentage of 1000 bootstrap replicates. (PDF $680 \mathrm{~kb}$ )

Additional file 3: Figure S2. Neighbour-joining phylogenetic tree of the partial pol gene. The scale bar indicates 5\% nucleotide sequence divergence. Values on the branches represent the percentage of 1000 bootstrap replicates. (PDF $719 \mathrm{~kb}$ )

Additional file 4: Figure S3. Neighbour-joining phylogenetic tree of the partial env gene. The scale bar indicates 10\% nucleotide sequence divergence. Values on the branches represent the percentage of 1000 bootstrap replicates. (PDF $591 \mathrm{~kb}$ )

Additional file 5: Table S2. The constituent of subjects successfully genotyped. (PDF $159 \mathrm{~kb}$ )

Additional file 6: Table S3. The proportion of CRF08_BC in men and women with different transmission routes. (PDF $68 \mathrm{~kb}$ )

Additional file 7: Table S4. The distribution of recent HIV infections was attributed to the main transmission routes in each prefecture. (PDF $67 \mathrm{~kb}$ )

\section{Abbreviations}

BED-CEIA: BED-capture enzyme immunoassay; CRF: Circulating recombinant form; FSW: Female sexual worker; HIV-1: Human immunodeficiency virus-1; IDU: Intravenous drug user; MSM: Men who have sex with men;

NNRTIs: Non-nucleoside reverse transcriptase inhibitors; NRTIs: Nucleoside reverse transcriptase inhibitors; PIs: Protease inhibitors; SDRMs: Surveillance drug resistance mutations; TDR: Transmitted drug resistance; URF: Unique recombinant form

\section{Acknowledgements}

We acknowledge the staff at the local Centers for Disease Control and Prevention for their assistance in sample collection.

\section{Authors' contributions}

Conceived and designed the experiments: MC, YM, ZS and MJ. Collected the samples: MC, HC, JD, CY, LD, XJ, MY and LY. Performed the experiments: MC, HC, JD, CY, LD, XJ, MY and LY. Collected epidemiological data: YM, HL, LS, ZS and MJ. Analysed the data: MC. Wrote the paper: MC. Approved the final version of this manuscript: MC, YM, HC, JD, HL, CY, LD, XJ, MY, LY, LS, ZS and MJ.

\section{Funding}

This work was supported by the National Natural Science Foundation of China (81560327) and the Research Project for Yunnan Science and Technology (2015FB200). The funders had no role in the study design, data collation and analysis, interpretation of data, or preparation of the manuscript.

\section{Availability of data and materials}

The sequences obtained in this study were submitted to NCBI GenBank under accession numbers MH572689-MH573900. The datasets used and/or 
analysed during the current study available from the corresponding author on reasonable request.

\section{Ethics approval and consent to participate}

This study was approved by the Biomedical Ethics Review Committee of Yunnan Province. The adults' written consents were provided by themselves. The juveniles' consents for HIV testing were provided by their guardians, if they had HIV, written consents about this study for HIV control and prevention were further obtained from their guardians when informing testing results.

\section{Consent for publication}

Not applicable.

\section{Competing interests}

The authors declare that they have no competing interests.

\section{Received: 13 April 2019 Accepted: 10 July 2019}

Published online: 29 July 2019

\section{References}

1. Lu L, Jia M, Ma Y, Yang L, Chen Z, Ho DD, et al. The changing face of HIV in China. Nature. 2008:455(7213):609-11.

2. Jia M, Luo H, Ma Y, Wang N, Smith K, Mei J, et al. The HIV epidemic in Yunnan Province, China, 1989-2007. J Acquir Immune Defic Syndr. 2010; 53(Suppl 1):S34-40.

3. National Health and Family Planning Commission of the People's Republic of China. 2015 China AIDS Response Progress Report. 2015.

4. Wu Z, Sullivan SG, Wang Y, Rotheram-Borus MJ, Detels R. Evolution of China's response to HIV/AIDS. Lancet. 2007;369(9562):679-90.

5. Graf M, Shao Y, Zhao Q, Seidl T, Kostler J, Wolf H, et al. Cloning and characterization of a virtually full-length HIV type 1 genome from a subtype B'-Thai strain representing the most prevalent B-clade isolate in China. AIDS Res Hum Retrovir. 1998;14(3):285-8.

6. Luo CC, Tian C, Hu DJ, Kai M, Dondero T, Zheng X. HIV-1 subtype C in China. Lancet. 1995;345(8956):1051-2.

7. Shao Y, Zhao Q, Wang B, Chen Z, Su L, Zeng Y, et al. Sequence analysis of HIV env gene among HIV infected IDUs in Yunnan epidemic area of China. Chin J Virol. 1994;10(4):291-9.

8. Cheng H, Zhang J, Capizzi J, Young NL, Mastro TD. HIV-1 subtype E in Yunnan, China. Lancet. 1994;344(8927):953-4.

9. Feng $Y$, Takebe $Y$, Wei H, He X, Hsi JH, Li Z, et al. Geographic origin and evolutionary history of China's two predominant HIV-1 circulating recombinant forms, CRF07_BC and CRF08_BC. Sci Rep. 2016;6:19279.

10. Tee KK, Pybus OG, Li XJ, Han X, Shang H, Kamarulzaman A, et al. Temporal and spatial dynamics of human immunodeficiency virus type 1 circulating recombinant forms 08_BC and 07_BC in Asia. J Virol. 2008;82(18):9206-15.

11. Yao $Y$, Wang $N$, Chu J, Ding $G$, Jin $X$, Sun $Y$, et al. Sexual behavior and risks for HIV infection and transmission among male injecting drug users in Yunnan, China. Int J Infect Dis. 2009;13(2):154-61.

12. Chen $M$, Yang L, Ma Y, Su Y, Yang C, Luo H, et al. Emerging variability in HIV-1 genetics among recently infected individuals in Yunnan, China. PLoS One. 2013:8(3):e60101.

13. Chen M, Ma Y, Su Y, Yang L, Zhang R, Yang C, et al. HIV-1 genetic characteristics and transmitted drug resistance among men who have sex with men in Kunming, China. PLoS One. 2014;9(1):e87033.

14. Chen M, Ma Y, Chen H, Dai J, Dong L, Yang C, et al. HIV-1 genetic transmission networks among men who have sex with men in Kunming, China. PLoS One. 2018;13(4):e0196548.

15. Han X, An M, Zhang W, Cai W, Chen X, Takebe Y, et al. Genome sequences of a novel HIV-1 circulating recombinant form, CRF55_01B, identified in China. Genome Announc. 2013;1:1.

16. Han X, An M, Zhang W, Zhao B, Chu Z, Takebe Y, et al. Genome sequences of a novel HIV-1 circulating recombinant form (CRF59_01B) identified among men who have sex with men in northeastern China. Genome Announc. 2013:1:3.

17. Wei $H$, Xing $H$, Hsi JH, Jia M, Feng $Y$, Duan $S$, et al. The sexually driven epidemic in youths in China's southwestern border region was caused by dynamic emerging multiple recombinant HIV-1 strains. Sci Rep. 2015;5:11323.
18. Miao J, Ran J, Song Y, Liu Y, Gao L, Miao Z, et al. Characterization of a novel HIV-1 circulating recombinant form, CRF01_AE/B'/C (CRF96_cpx), in Yunnan, China. AIDS Res Hum Retrovir. 2018;34(4):393-7.

19. Li Y, Miao J, Miao Z, Song Y, Wen M, Zhang Y, et al. Identification of a novel HIV type 1 circulating recombinant form (CRF86_BC) among heterosexuals in Yunnan, China. AIDS Res Hum Retrovir. 2017;33(3):279-83.

20. Hu Y, Wan Z, Zhou YH, Smith D, Zheng YT, Zhang C. Identification of two new HIV-1 circulating recombinant forms (CRF87_cpx and CRF88_BC) from reported unique recombinant forms in Asia. AIDS Res Hum Retrovir. 2017; 33(4):353-8.

21. Song $Y$, Feng $Y$, Miao Z, Wang B, Yang M, Zhang AM, et al. Near-fulllength genome sequences of a novel HIV-1 circulating recombinant form, CRF01 AE/B'/C (CRF78 cpx), in Yunnan, China. AIDS Res Hum Retrovir. 2016;32(6):601-6.

22. Wei $H$, Liu Y, Feng Y, Hsi J, Xing H, He X, et al. Genome sequence of a novel HIV-1 circulating recombinant form (CRF57_BC) identified from Yunnan, China. AIDS Res Hum Retrovir. 2014;30(4):384-8.

23. Wei H, His J, Feng Y, Xing H, He X, Liao L, et al. Identification of a novel HIV1 circulating recombinant form (CRF62_BC) in western Yunnan of China. AIDS Res Hum Retrovir. 2014;30(4):380-3.

24. Hsi J, Wei $H$, Xing $H$, Feng $Y$, He X, Liao L, et al. Genome sequence of a novel HIV-1 circulating recombinant form (CRF64_BC) identified from Yunnan, China. AIDS Res Hum Retrovir. 2014;30(4):389-93.

25. Feng $Y$, Wei H, Hsi J, Xing H, He X, Liao L, et al. Identification of a novel HIV type 1 circulating recombinant form (CRF65_cpx) composed of CRF01_AE and subtypes B and C in Western Yunnan, China. AIDS Res Hum Retrovir. 2014;30(6):598-602.

26. Tamura K, Stecher G, Peterson D, Filipski A, Kumar S. MEGA6: molecular evolutionary genetics analysis version 6.0. Mol Biol Evol. 2013;30(12): 2725-9.

27. Kulldorff M. and Information Management Services, Inc. SaTScan ${ }^{\mathrm{TM}}$ v9.6: Software for the spatial and space-time scan statistics, 2018 www.satscan.org.

28. Wand H, Ramjee G. Targeting the hotspots: investigating spatial and demographic variations in HIV infection in small communities in South Africa. J Int AIDS Soc. 2010;13:41.

29. Gonzalez R, Augusto OJ, Munquambe K, Pierrat C, Pedro EN, Sacoor C, et al. HIV incidence and spatial clustering in a rural area of southern Mozambique. PLoS One. 2015;10(7):e0132053.

30. Cuadros DF, Abu-Raddad L. Spatial variability in HIV prevalence declines in several countries in sub-Saharan Africa. Health Place. 2014;28:45-9.

31. Hixson BA, Omer SB, del Rio C, Frew PM. Spatial clustering of HIV prevalence in Atlanta, Georgia and population characteristics associated with case concentrations. J Urban Health. 2011;88(1):129-41.

32. Wylie JL, Cabral T, Jolly AM. Identification of networks of sexually transmitted infection: a molecular, geographic, and social network analysis. J Infect Dis. 2005;191(6):899-906.

33. Jennings JM, Curriero FC, Celentano D, Ellen JM. Geographic identification of high gonorrhea transmission areas in Baltimore, Maryland. Am J Epidemiol. 2005;161 (1):73-80.

34. Acharya BK, Cao C, Lakes T, Chen W, Naeem S. Spatiotemporal analysis of denque fever in Nepal from 2010 to 2014. BMC Public Health. 2016;16(1):849.

35. Quantum GIS Development Team, Quantum GIS Geographic Information System. Open Source Geospatial Foundation Project, 2002, http://www.qgis.org.

36. Gifford RJ, Liu TF, Rhee SY, Kiuchi M, Hue S, Pillay D, et al. The calibrated population resistance tool: standardized genotypic estimation of transmitted HIV-1 drug resistance. Bioinformatics. 2009; 25(9):1197-8

37. Chen M, Jia MH, Ma YL, Luo HB, Chen HC, Yang CJ, et al. The changing HIV-1 genetic characteristics and transmitted drug resistance among recently infected population in Yunnan, China. Epidemiol Infect. 2018;146(6):775-81.

38. Pang W, Zhang C, Duo L, Zhou YH, Yao ZH, Liu FL, et al. Extensive and complex HIV-1 recombination between B', C and CRF01_AE among IDUs in south-East Asia. AIDS. 2012;26(9):1121-9.

39. Chen $X$, Ye M, Duo L, Pang W, Smith D, Zhang C, et al. First description of two new HIV-1 recombinant forms CRF82_cpx and CRF83_cpx among drug users in northern Myanmar. Virulence. 2017:8(5):497-503.

40. Su L, Wei D, Yang H, Zeng Y, Hu Y, Yuan D, et al. Identification of a novel HIV-1 circulating recombinant form (CRF85_BC) in Sichuan, China. AIDS Res Hum Retrovir. 2016;32(9):895-9. 
41. Chen $X$, Zhou YH, Ye M, Wang Y, Duo L, Pang W, et al. Burmese injecting drug users in Yunnan play a pivotal role in the cross-border transmission of HIV-1 in the China-Myanmar border region. Virulence. 2018;9(1):1195-204

42. Chen $X$, Duo L, Ye M, Zhang C, Zheng YT. Non-Chinese immigrants: challenge faced by Yunnan of China to achieve the 90-90-90 goals. Virol Sin. 2018;33(3):291-3.

\section{Publisher's Note}

Springer Nature remains neutral with regard to jurisdictional claims in published maps and institutional affiliations.

- fast, convenient online submission

- thorough peer review by experienced researchers in your field

- rapid publication on acceptance

- support for research data, including large and complex data types

- gold Open Access which fosters wider collaboration and increased citations

- maximum visibility for your research: over $100 \mathrm{M}$ website views per year

At BMC, research is always in progress. 